\title{
Carrier Transport Properties of CdTe Detector under Polarization Condition
}

\author{
Hisaya Nakagawa, ${ }^{1 *}$ Tsuyoshi Terao, ${ }^{1}$ Tomoaki Masuzawa, ${ }^{2}$ Tetsu Ito, ${ }^{2}$ \\ Akifumi Koike, ${ }^{2,3}$ Hisashi Morii, ${ }^{2,3}$ and Toru Aoki ${ }^{1,2,3}$ \\ ${ }^{1}$ Graduate Schoool of Science and Technology, Shizuoka University, \\ 3-5-1 Johoku, Nakaku, Hamamatsu, Shizuoka 432-8011, Japan \\ ${ }^{2}$ Reserch Institute of Electronics, Shizuoka University, \\ 3-5-1 Johoku, Nakaku, Hamamatsu, Shizuoka 432-8011, Japan \\ ${ }^{3}$ ANSeeN Inc., 3-5-1 Johoku, Nakaku, Hamamatsu, Shizuoka 432-8011, Japan
}

(Received February 1, 2018; accepted April 25, 2018)

Keywords: radiation detector, semiconductor detector, $\mathrm{CdTe}$, polarization, carrier transport properties

Cadmium telluride (CdTe) is a promising material for semiconductor-based roomtemperature radiation detectors because of its wide bandgap and relatively good carrier transport properties. One remaining issue of CdTe detectors is an instability arising in longterm operation. This instability, called polarization, is explained by charge accumulation due to carrier trapping/detrapping at deep defect levels. However, previous explanations did not include carrier transport in CdTe. In this study, we have investigated carrier transport properties by measuring the carrier transit time at each stage of polarization. As a result, we have developed a polarization model including carrier transport and calculated the electric field distribution across the CdTe detector during polarization.

\section{Introduction}

Cadmium telluride (CdTe) is considered to be a suitable material for semiconductor-based $\mathrm{X}$-ray and gamma-ray detectors. ${ }^{(1-3)}$ CdTe-based detectors have high X-/gamma-ray detection efficiencies because CdTe is composed of high-atomic-number elements and have high density. Moreover, CdTe can realize low thermal noise operation at room temperature owing to its relatively wide bandgap of $\sim 1.4 \mathrm{eV}$. Potential applications of such detectors range from medical and dental radiography, computed tomography, to nondestructive inspection.

Despite these advantages, the application of CdTe-based radiation detectors is limited to relatively short time operations; a severe degradation of detector performance is known in long-term operation and is called polarization. ${ }^{(1)}$ The degradation caused by polarization includes a decrease in peak count and the shifting of the peak energy over operation time. The polarization is often explained using the charge accumulation model. ${ }^{(4-6)}$ In this model, the polarization phenomenon is explained by carrier trapping/detrapping at deep acceptor levels. Electrons trapped in the deep acceptor levels act as negative space charges and alter the potential

*Corresponding author: e-mail: nakagawa.hisaya.14@shizuoka.ac.jp http://dx.doi.org/10.18494/SAM.2018.1929 
distribution across the CdTe detector. As the charges build up, the depletion layer width in CdTe decreases, which prevents the collection of signal charges. However, the ohmic CdTe detector has not been reported. ${ }^{(7)}$ Therefore, many mechanisms have been suggested to explain polarization and the details have been discussed. ${ }^{(4-9)}$ In a previous study, a modified model of polarization was suggested from current-voltage $(I-V)$ characteristics by Toyama et al. ${ }^{(8)}$ Others measured the electric field distribution in the detector under polarization conditions, utilizing the Pockels effect. ${ }^{(10-12)}$ However, few studies have focused on carrier transport and collection during polarization.

In this study, we measured carrier transport properties during polarization by measuring the pulse height and carrier transit time of signal charges. For this purpose, we developed a novel measurement system that enables the simultaneous measurement of both pulse height and carrier transit time. ${ }^{(13)}$ The carrier transport properties obtained using this system were applied to the previous charge accumulation model ${ }^{(8)}$ to estimate the electric field distribution in the CdTe detector to investigate the validity of this model.

\section{Materials and Methods}

The principle of radiation detection in our system is described as follows. When radiation is absorbed in the CdTe detector, multiple electron-hole pairs are generated. The generated carriers are driven by the electric field in the CdTe detector and collected at the electrodes of the detector. The collected charges are converted to a signal pulse by a charge-sensitive amplifier (CSA), and the pulse signals are analyzed with a signal processing system. Figure 1 shows the output pulse signal from the CSA. The height of the pulse signal is proportional to the incident radiation energy, and the pulse rise time indicates the time needed to collect signal carriers. The signal processing system is designed to measure both the height and rise time of the signal pulses simultaneously, so that carrier transit time can be measured at each degree of polarization.

The CdTe detectors used in this study were Schottky diodes based on a p-type crystal fabricated by the travelling heater method (THM) at Acrorad Co., Ltd. Indium (In) was deposited on one side of the CdTe detector to form a Schottky electrode, and platinum (Pt)

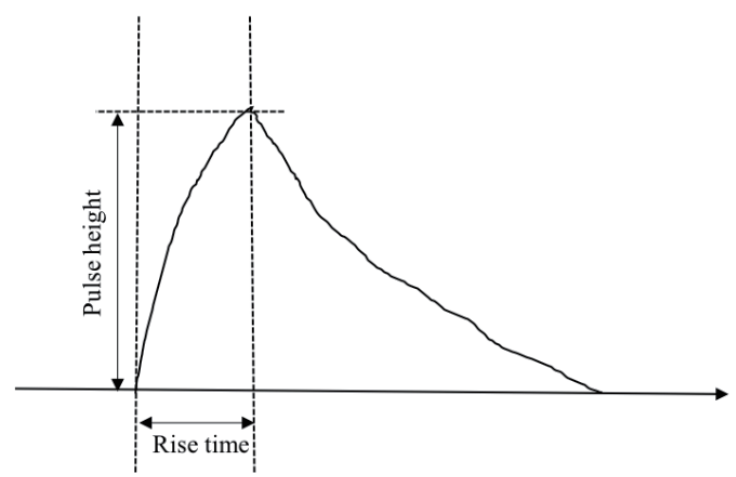

Fig. 1. Schematic diagram of signal pulse from CSA. The novel signal processing system can yield the pulse height and rise time as energy and carrier transit time, respectively. 
was deposited on the other side of the detector to serve as an ohmic electrode. The size of the CdTe detector was $4 \times 4 \mathrm{~mm}^{2}$ and the thickness of CdTe was $0.5 \mathrm{~mm}$. The CdTe detector was connected to the CSA (CLEAR-PULSE Co., Ltd., Model 5102) and the output signals from the CSA were introduced to a specially designed signal processing system (ANSeeN Inc. ANSHSMCA1-2D).

The measurement is conducted as follows. A reverse bias of $100 \mathrm{~V}$ is applied to the detector to obtain the energy spectrum of a gamma-ray from ${ }^{241} \mathrm{Am}$ radioisotopes. The detector is operated for over $6 \mathrm{~h}$ to observe the progress of polarization over time. At the beginning, the detector is assumed to be fully depleted by the applied bias. With time, polarization occurs and the gamma-ray spectra measured by the detector show peak shifts and a reduction in energy resolution. The energy spectra of gamma-rays are obtained every $60 \mathrm{~min}$ to evaluate the effect of polarization over time. It should be noted that each gamma-ray spectrum is observed in 5 min so that the polarization condition does not change during each measurement.

In this study, gamma-rays from the ${ }^{241} \mathrm{Am}$ radioisotope $(59.5 \mathrm{keV})$ were measured at room temperature. The gamma-rays from ${ }^{241} \mathrm{Am}$ have relatively low energies and are mostly absorbed near the surface of the CdTe detector. By irradiating either side of the CdTe detector, we can select which carriers (electrons or holes) are transported longer in the CdTe detector. When the In-deposited side of the detector is irradiated with gamma-rays, electrons are read out immediately, and holes drift across the detector before being collected at the other side of the detector. On the other hand, when the Pt-deposited side is irradiated, electrons drift across the detector before being collected as the signal. Therefore, we can evaluate the carrier transport properties of both electrons and holes by changing the irradiation conditions.

\section{Results and Discussion}

Figure 2 shows energy spectra of gamma-rays from ${ }^{241} \mathrm{Am}$, obtained every $60 \mathrm{~min}$. The pulse height of radiation signals is converted to channel number, which corresponds to the energy of the incident gamma-ray. Figure 2(a) shows the spectra obtained by irradiating the

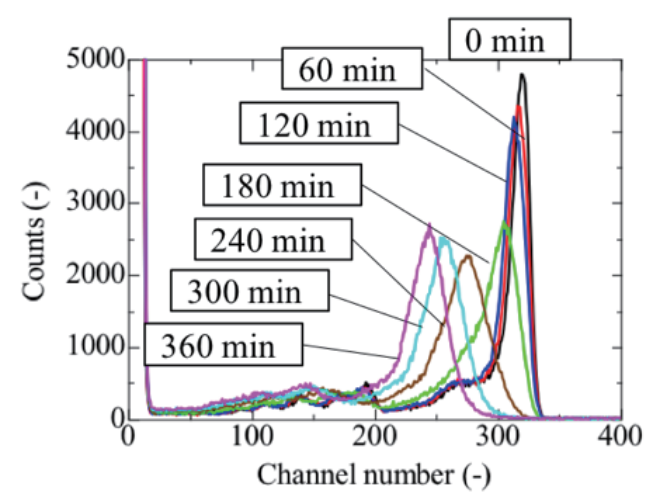

(a)

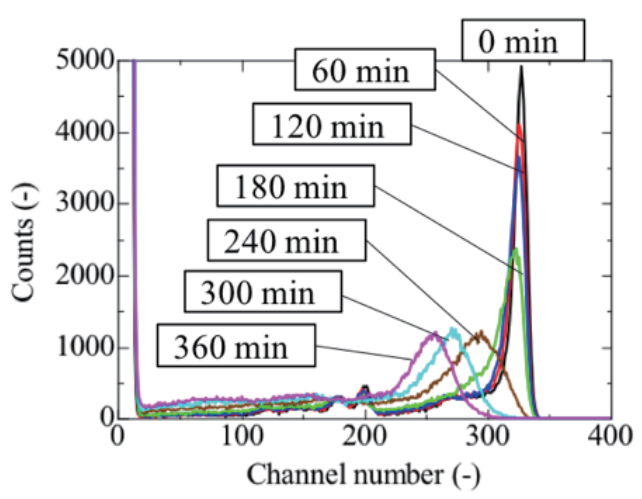

(b)

Fig. 2. (color online) Pulse height spectrum for ${ }^{241} \mathrm{Am}$ irradiation from (a) In-deposited side (anode side) and (b) Pt-deposited side (cathode side) with time. The channel number at the start of bias voltage application (0 min) corresponds to the ${ }^{241} \mathrm{Am}$ photoelectric peak energy of $59.5 \mathrm{keV}$. 
gamma-rays from the In-deposited side of the CdTe detector, and Fig. 2(b) shows the spectra with gamma-rays irradiated from the Pt-deposited side. It was confirmed that the peaks of ${ }^{241} \mathrm{Am}$ gamma-rays shifted to a lower channel number (i.e., energy) and the counts of ${ }^{241} \mathrm{Am}$ peaks decreased with time. These features are characteristic of a detector suffering from polarization. It was also confirmed that the effect of polarization became more significant as the device was operated longer. Figure 3 shows the carrier transit time of the signal pulses for the main peak (peak due to photoelectric absorption) after each measurement interval. Here, the transit time represented the rise time of the output signal pulse from the CSA. In the case of In-deposted side irradiation, the carrier transit time did not change up to $120 \mathrm{~min}$. However, the carrier transit time increased after $120 \mathrm{~min}$. With an increase in operation time, the carrier transit time began to decrease. A similar result was obtained for Pt-deposited side irradiation. No decrease in transit time beyond $180 \mathrm{~min}$ was expected. A possible explanation is as follows: at the early stage of polarization, electric field accumulates at the surface owing to the space charge, and the electric field across the CdTe detector weakens. This results in a longer carrier transit time. As the polarization advances, more space charges accumulate, and at some point, the width of the depletion layer begins to decrease. Since the electric field exists only in the depletion layer, carriers generated outside the depletion layer cannot be read out as a signal, and those generated in the depletion layer will be read out. This results in a smaller carrier transport length, and thus carrier transit time is shortened. The change in carrier transit time suggests that the polarization not only changes the intensity of electric field but also reduces the width of the depletion layer.

Since the reduction in depletion layer width was expected, we evaluated the depletion layer width by integrating the peak count. The number of detected gamma-ray photons was proportional to the depletion layer width. Figure 4 shows the change in the integrated count of photoelectric peaks obtained from the energy spectra of irradiation on each side. When ${ }^{241} \mathrm{Am}$ gamma-rays were irradiated on the In-deposited side of the CdTe detector, the integrated peak number did not change with time. This result means that ${ }^{241} \mathrm{Am}$ gamma-rays are absorbed near the In-deposited side and cannot reach the Pt-deposited side of the CdTe detector. On the other hand, in the case of Pt-deposited side irradiation, the integrated count started to decrease after

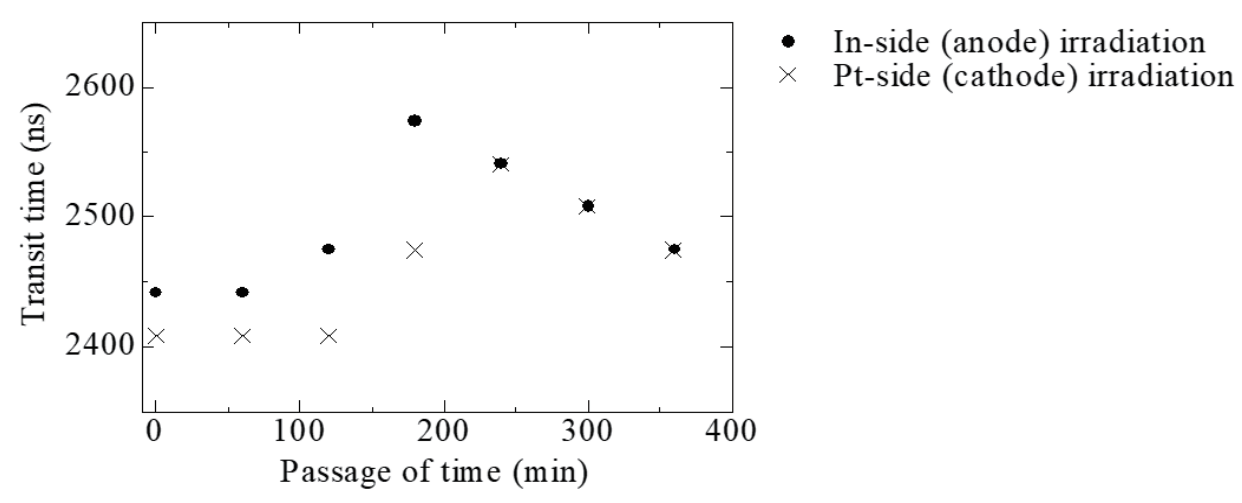

Fig. 3. Transit time upon ${ }^{241} \mathrm{Am}$ irradiation from each side with time. Circles correspond to In-deposited side irradiation and crosses to Pt-deposited side irradiation. 


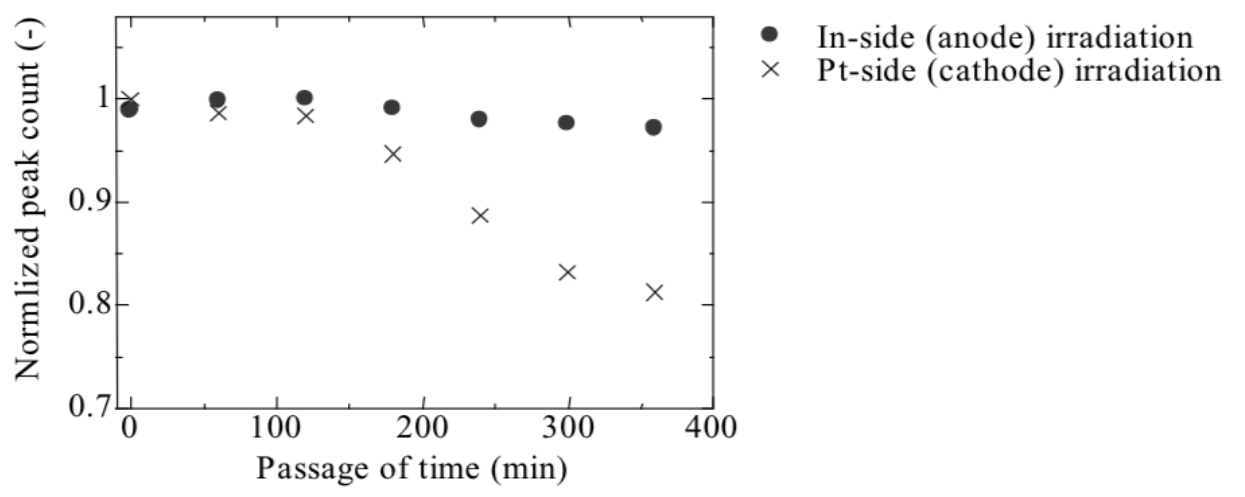

Fig. 4. Changes in counts of ${ }^{241} \mathrm{Am}$ photoelectric peaks upon In-and Pt-deposited side irradiations. Circles correspond to In-deposited side irradiation and crosses to Pt-deposited side irradiation.

$120 \mathrm{~min}$ and was reduced by about $20 \%$ after $360 \mathrm{~min}$. From the change in integrated peak count, the depletion layer thickness was confirmed to decrease with time and the dead layer for interaction was formed from the Pt-deposited side of the CdTe detector.

From these results, we estimated the polarization mechanism. The initial state is defined by the depletion layer being fully formed in the CdTe detector, and the generated carriers can fully be collected. When polarization begins, the potential of the CdTe detector is contorted by the accumulated space charges. The electric field is changed by the accumulated charges, and the drift velocity decreases. As the polarization proceeds further, the dead layer is formed and the depletion layer width decreases. For these reasons, the carrier transit time becomes shorter than the initial state and carriers are not collected sufficiently. To compare our estimation model with the existing model, the electric field distributions of the modified charge accumulation model suggested by Toyama et al. ${ }^{(8)}$ were calculated as

$$
\varepsilon(x, t)= \begin{cases}-\frac{q N_{T}}{\epsilon} x+\frac{q N_{T}}{2 \epsilon D}\left[D^{2}-(D-\lambda)^{2} \exp \left(-\frac{t}{\tau}\right)\right]+\frac{V_{R}}{D}, & 0 \leq x \leq \lambda \\ -\frac{q N_{T}}{\epsilon}\left[1-\exp \left(-\frac{t}{\tau}\right)\right] x+\frac{q N_{T}}{2 \epsilon D}\left[D^{2}-\left(D^{2}+\lambda^{2}\right) \exp \left(-\frac{t}{\tau}\right)\right]+\frac{V_{R}}{D}, & \lambda \leq x \leq D\end{cases}
$$

where $q$ is the magnitude of electric charge, $N_{T}$ is the total concentration of deep acceptors, $\epsilon$ is the dielectric constant of the semiconductor $\left(\epsilon=10.2 \epsilon_{0}\right.$ for CdTe, where $\epsilon_{0}$ is the dielectric constant in vacuum), $x$ is the position from the interface of the Schottky contact, $D$ is the thickness of the CdTe detector, $\tau$ is the detrapping time, $t$ is the operation time, $V_{R}$ is the reverse bias voltage, and $\lambda$ is the point of intersection between the deep acceptor level and the Fermi level. Moreover, the carrier transit time was calculated from this distribution. Figure 5 shows the carrier transit time derived using the modified charge accumulation model. The calculated carrier transit time also gradually increases with time. In addition, the carrier transit time decreases with increasing operation time. This result indicates almost the same tendency as that of the carrier transit time obtained by our measurement. 


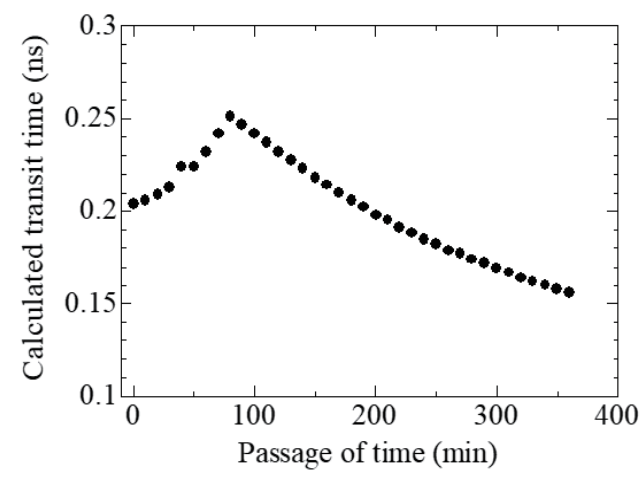

Fig. 5. Transit time calculated using charge accumulation model.

\section{Conclusions}

In this study, we measured changes in carrier transport properties during polarization, by measuring both the pulse height and rise time of a signal pulse using a newly developed signal processing system. The effect of polarization was confirmed by the shifted peak energy, loss of energy resolution, and the decrease in peak intensity in the energy spectra of gamma-rays from ${ }^{241} \mathrm{Am}$. During polarization, the carrier transit time first became longer, but then became shorter with time. This unexpected tendency was explained by the combination of a decrease in electric field across the CdTe detector and a decrease in depletion layer width. From these results, we estimated the mechanisms of polarization from carrier transport properties. The electric field distribution was calculated using the model, which showed good agreement with the measured carrier transit time and previous charge accumulation model. ${ }^{(8)}$

\section{Acknowledgments}

This research is based on the Cooperative Research Project of Research Center for Biomedical Engineering/Research Institute of Electronics, Shizuoka University.

\section{References}

1 M. Niraula A. Nakamura, T. Aoki, Y. Tomita, and Y. Hatanaka: Nucl. Instrum. Methods Phys. Res., Sect. A 491 (2002) 168.

2 Y. Eisen and A. Shor: J. Cryst. Growth (1998) 1302.

3 C. Scheirber: Nucl. Instrum. Methods Phys. Res., Sect. A 448 (2000) 513.

4 H. L. Malm and M. Martini: IEEE Trans. Nucl. Sci. 21 (1974) 322.

5 R. O. Bell, G. Entine, and H. B. Serreze: Nucl. Instrum. Methods 117 (1974) 267.

6 P. Shiffert, J. Berger, C. Scharager, A. Cornet, and R. Stuck: IEEE Trans. Nucl. Sci. 23 (1976) 159.

7 K. Okada, Y. Sakurai, and H. Suematsu: Appl. Phys. Lett. 90 (2007) 063504.

8 H. Toyama, A. Higa, M. Yamazato, T. Maehara, R. Ohno, and M. Toguchi: Jpn. J. Appl. Phys. 45 (2006) 8842.

9 R.Grill, E. Belas, J. Franc, M. Bugar, S. Uxa, P. Moravec, and P. Hoschl: IEEE Trans. Nucl. Sci. 58 (2011) 3172.

10 J. Franc, V. Dedic, P. J. Sellin, R. Grill, and P. Veeramani: Appl. Phys. Lett. 98 (2011) 232115.

11 I. Farella, G. Montagna, A. M. Mancini, and A. Cola: IEEE Trans. Nucl. Sci. 56 (2009) 1736.

12 A. Cola and I Farella: Appl. Phys. Lett. 94 (2009) 102113.

13 A. Koike, T. Okunoyama, T. Ito, H. Morii, Y. Neo, H. Mimura, and T. Aoki: IEEE Nucl. Sci. Symp. Conf. Rec. (NSS/MIC) (IEEE, 2010) 3797. 